\title{
Corela
}

Cognition, représentation, langage

HS-3 | 2006

Les verbes d'apparence

\section{La pièce fait vide, Das Zimmer wirkt leer.Plaidoyer pour un petit système clos de l'apparence en allemand.}

\section{Alain Cambourian}

\section{OpenEdition}

Journals

Édition électronique

URL : http://journals.openedition.org/corela/1268

DOI : 10.4000/corela. 1268

ISSN : 1638-573X

Éditeur

Cercle linguistique du Centre et de l'Ouest - CerLICO

Référence électronique

Alain Cambourian, « La pièce fait vide, Das Zimmer wirkt leer.Plaidoyer pour un petit système clos de l'apparence en allemand. », Corela [En ligne], HS-3 | 2006, mis en ligne le 13 mars 2006, consulté le 02 mai 2019. URL : http://journals.openedition.org/corela/1268 ; DOI : 10.4000/corela.1268

Ce document a été généré automatiquement le 2 mai 2019.

\section{(c) (i) (2)(2)}

Corela - cognition, représentation, langage est mis à disposition selon les termes de la licence Creative Commons Attribution - Pas d'Utilisation Commerciale - Partage dans les Mêmes Conditions 4.0 International. 


\title{
La pièce fait vide, Das Zimmer wirkt leer.Plaidoyer pour un petit système clos de l'apparence en allemand.
}

\author{
Alain Cambourian
}

1 Le champ d'observation rassemble des éléments extrêmement divers et variés si l'on prend comme point de départ les équivalents allemands des termes français «sembler», «paraître», «apparaittre», "avoir l'air», «faire» ou encore «ressembler» présentés par Alain Delplanque dans son introduction à la présente étude. Quelques exemples autour de «faire», extraits du Dictionnaire français-allemand Harrap's Weis Mattutat et du Duden Universalwörterbuch :

(1) Il fait le clown : er spielt den Clown - où le verbe «spielen» est celui qui signifie par ailleurs «jouer».

(2) Il fait le sourd: er stellt sich taub / er tut so, als sei er taub - où le verbe «stellen» est celui qui signifierait dans un autre contexte «se mettre (debout)», et le verbe «tun» celui qui signifie «faire» (cf. anglais «do»).

(3) Il fait jeune : er sieht jung aus - «aussehen» signifiant «avoir l'air».

(4) Il fait riche : er macht den Eindruck, als sei er reich - avec l'idée de «faire» (machen) une certaine «impression» (Eindruck).

(5) Il fait moderne : er wirkt modern - où «wirken», de la famille de «Werk», œuvre, ouvrage, apporte l'idée d'un impact, d'un effet produit, bien ancré dans la réalité.

2 Ces quelques exemples suffisent à dégager deux directions, deux perspectives en quelque sorte :

- la volonté du sujet observé de donner l'apparence de quelque chose (cf. français «faire semblant»);

- la perception de l'apparence par un observateur / locuteur / énonciateur extérieur.

Choisissant de me limiter ici à cette dernière, c'est-à-dire en considérant la volonté du sujet comme non pertinente dans cette étude, j'obtiens par une commutation toute simple, à partir de la représentation d'une «pièce vide», le paradigme suivant :

(6) Das Zimmer ist leer. 
(7) Das Zimmer bleibt leer.

(8) Das Zimmer steht leer.

(9) Das Zimmer wirkt leer.

(10) Das Zimmer sieht leer aus.

(11) Das Zimmer scheint leer.

(12) Das Zimmer erscheint leer.

\section{Le système et sa syntaxe}

4 Dans le paradigme ainsi obtenu, le troisième noyau verbal tient une place un peu particulière, car le sens «ist nicht bewohnt / est inoccupée» qui résulte de la combinaison de «steh-» et de «leer» donne à cette expression le statut de lexie. Les deux premiers noyaux verbaux - auxquels il convient d'ajouter «werden» qui fonctionne certes mal dans ce contexte précis, mais qui fonctionnerait sans problème dans d'autres - servent à exprimer l'attribution d'une qualité : la qualité «leer» est dans les deux cas attribuée à «das Zimmer».

5 Avec les quatre derniers noyaux verbaux en revanche, l'attribution de la qualité «leer» n'est qu'apparente, car elle passe par le filtre d'un observateur. Il n'y a donc pas, dans ce dernier cas, à proprement parler «attribution» d'une quelconque qualité, la qualité dont il s'agit est en quelque sorte mise en attente : en attente d'infirmation ou de confirmation. Il est symptomatique de constater en effet que ces quatre noyaux verbaux ont en commun - et c'est avec la mention de la qualité la seule structure qu'ils partagent tous les quatre - la possibilité de se combiner avec «als ob + groupe verbal», c'est-à-dire que dans la démarche d'identification (als) à un autre monde, une alternative (ob) est présentée, qui associe le contenu non actualisé d'une proposition à sa contradictoire : "p oder non p», c'est oui ou c'est non? Exemples (13) à (16) :

(13) Der Inhalt wirkt, als ob er auf einer Pyramide dargestellt werden würde.

Le texte à l'intérieur [il s'agit de la valeur groove attachée à un style de cadre] fait l'effet d'être présenté sur une pyramide.

(14) Die Fabrik sieht aus, als ob sie ein Teil dieser schönen Landschaft wäre.

L'implantation de cette usine à cet endroit lui donne l'air d'être partie intégrante de ce beau paysage.

(15) Es scheint, als ob Sie keine Formation hätten.

Il semble que vous n'ayez aucune formation.

(16) Frage: Es erscheint, als ob bei einer bestimmten Person dieselben Gedanken in das Bewußtseinsfeld treten. Antwort: Ja, daraus entsteht die Person, und daraus besteht sie: aus einer gewissen gewohnheitsmäßigen Art zu denken.

Question: Les choses se présentent comme si pour une personne donnée c'étaient toujours les mêmes pensées qui pénètrent dans le champ de la conscience. Réponse : Oui, c'est bien ce qui donne naissance à la personne, et ce dont elle tire son existence : une certaine façon habituelle de penser.

Tous exemples extraits de textes réels via Google.

C'est pourquoi, tout en reconnaissant le lien fonctionnel qu'entretiennent ces quatre noyaux verbaux avec les noyaux bien connus d'attribution par leur appartenance au même paradigme, je plaiderai, pour l'allemand, pour la définition d'un petit sous-système clos de l'apparence. 


\section{2. Éléments de sémantisme}

8 Une bipartition peut être opérée d'emblée à l'intérieur de ce sous-système, en faisant réagir les quatre noyaux verbaux qui le constituent à la présence dans leur contexte d'un datif tel que «mir»: deux d'entre eux acceptent cette présence, deux autres ne la supportent pas. Exemples (17) à (20) :

(17) *Das Zimmer wirkt mir leer.

(18) *Das Zimmer sieht mir leer aus.

(19) Das Zimmer scheint mir leer.

(20) Das Zimmer erscheint mir leer.

9 La possibilité de la présence de «mir» signifie la possibilité de la présence explicite de l'observateur; son impossibilité signifie que la présence de ce dernier reste nécessairement implicite.

Conjointement, je constate : les deux verbes compatibles avec la présence de «mir» le sont également avec celle d'un groupe verbal introduit par «dass» et avec celle d'un infinitif précédé de «zu». Pas question d'imposer une telle présence aux deux autres! Exemples toujours Google :

11 Présence d'un groupe verbal introduit par «dass», exemples (21) à (24) :

(21) Genossen, es scheint, dass ihr die Arbeit niedergelegt habt.

Camarades! Il semble que vous ayez cessé le travail.

(22) Beim Öffnen der Admins erscheint «Server angehalten». Info: Sie öffnen die Admin[istrations]konsole von fax@NET Server und es erscheint, dass der Server gestoppt ist! Antwort: Dies ist absolut normal, immer wenn Sie die Adminkonsole öffnen, wird der Dienst beendet. Nachdem Sie Ihre Einstellung gemacht haben, und die Konsole mit «OK» wieder schliessen, werden alle Einstellungen gespeichert und der Dienst wird wieder gestartet.

Le message "Serveur hors de service» apparaît à l'ouverture du fichier «Administration». Information : Vous ouvrez la console «Administration»du serveur Fax@net et il apparait que le serveur est hors de service! Réponse : ceci est absolument normal, le service se termine chaque fois que vous ouvrez la console. Une fois que vous avez fait ce que vous aviez à faire et que vous avez refermé la console en cliquant sur «OK», tout ce que vous avez fait est enregistré et le service se remet en route.

(23a) Die wichtigste Eigenschaft [der Dirac'schen Delta-Funktion $d(x)$ ] ist, dass sie im Produkt $f(x) . d(x)$ mit einer anderen Funktion $f(x)$ so wirkt, dass auch alle Funktionswerte des Produkts gleich 0 werden, für alle $\mathrm{x}$ außer $\mathrm{x}=0$.

La propriété la plus importante de la fonction $\partial d(x)$ de Dirac est que la fonction résultant de son produit $f(x) \cdot d(x)$ avec une autre fonction $f(x)$ est nulle en dehors de l'origine et d'intégrale égale à 1 .

(23b) *[...] sie im Produkt $\mathrm{f}(\mathrm{x}) \mathrm{d}(\mathrm{x})$ mit einer anderen Funktion $\mathrm{f}(\mathrm{x})$ wirkt, dass auch alle Funktionswerte des Produkts gleich 0 werden [...].

(24a) Tja, es sieht so aus, dass in den nächsten $12 \mathrm{~h}$ entweder ein gigantischer Schneesturm mit $50 \mathrm{~cm}$ Neuschnee um die Ecke dampfen kann, oder eben auch gar rein nix.

Ouais! Ça peut aussi bien donner une gigantesque tempête de neige qui vous tombera dessus dans les douze heures qui viennent avec cinquante centimètres de neige fraîche, que rien $\mathrm{du}$ tout, mais alors rien du tout.

(24b) *Tja, es sieht aus, dass in den nächsten $12 \mathrm{~h}$ entweder ein gigantischer Schneesturm mit $50 \mathrm{~cm}$ Neuschnee um die Ecke dampfen kann [...]. 

«so» qui précède, élément bien connu comme anaphorique de qualité. La suppression de «so» dans les énoncés (23b) et (24b) entraîne l'agrammaticalité desdits énoncés.

Présence d'un infinitif précédé de «zu», exemples (25) à (28) :

(25) Der Mond scheint wesentlich größer zu sein, wenn er knapp über dem Horizont steht, als wenn er sich im Zenit befindet.

La Lune à l'horizon paraît sensiblement plus grande que celle au zénith.

(26) Dazu kommt noch folgendes Paradoxon: Der wegen des scheinbar größeren Abstands «am Horizont» größer wahrgenommene Mond erscheint dann wegen seiner ungewöhnlichen Größe wieder näher zu sein.

Vient s'ajouter à cela le paradoxe suivant : cette lune que nous percevons plus grande par suite de la plus grande distance apparente lorsqu'elle est à l'horizon nous semble alors être plus près de nous si l'on en juge par sa taille inhabituelle.

(27a) Zur scheinbaren Größenveränderung trägt auch das Prinzip der Vergleichsobjekte bei: Weil der Mond am Horizont im Vergleich mit kleineren Objekten, etwa Bäumen oder Häusern gesehen wird, wirkt er dort größer, als wenn er im Zenit im Vergleich mit dem großen Firmament gesehen wird.

Le principe de comparaison des objets entre eux joue également un rôle non négligeable dans l'apparente modification de la taille de la Lune : c'est parce que la Lune à l'horizon est vue par comparaison avec des objets plus petits, comme par exemple des arbres ou des maisons, qu'elle donne l'impression d'être plus grande dans cette position que lorsqu'elle est, au zénith, vue par comparaison avec le firmament infiniment grand.

(27b) *Weil der Mond am Horizont im Vergleich mit kleineren Objekten, etwa Bäumen oder Häusern gesehen wird, wirkt er dort größer zu sein, als wenn er im Zenit im Vergleich mit dem großen Firmament gesehen wird.

(28a) Warum sieht der Mond am Horizont größer aus, als wenn er hoch am Himmel steht? Das ist eine reine optische Täuschung, die auch als Mondillusion bekannt ist. Pourquoi la Lune à l'horizon a-t-elle l'air plus grande que lorsqu'elle est au plus haut du ciel ? Ce n'est rien d'autre qu'une illusion d'optique, bien connue sous le nom d'illusion de la Lune.

(28b) *Warum sieht der Mond am Horizont größer aus zu sein, als wenn er hoch am Himmel steht?

14 La possibilité de la présence d'un groupe infinitival signifie que le verbe est compatible avec une certaine aspectualité. Son impossibilité signifie que l'ensemble de l'énoncé est de valeur stative.

La possibilité de la présence d'un groupe verbal introduit par «dass» signifie que le verbe d'apparence repose sur un prédicat préconstruit, distancié par rapport à l'énonciateur. Son impossibilité signifie que l'énonciateur construit le prédicat directement à travers les apparences.

\subsection{Wirken}

Et en effet, «wirken», porteur de l'idée de "faire / produire un certain effet», est dans ce sous-système le noyau verbal qui convient, plutôt que «aussehen», pour traduire «La pièce fait vide», ainsi que je l'ai esquissé dans le titre du présent article. Un effet passant, implicitement puisque «mir» est interdit ici, par le filtre, le regard de l'observateur, comme en témoignent les exemples (29) à (32) :

(29) Über den Pool können wir nicht viel sagen, da wir nie dort waren, aber er wirkt gepflegt und die Duschen sind sehr schön.

De la piscine nous ne pouvons pas dire grand-chose, car nous n'y avons jamais été, mais elle fait l'effet d'une piscine bien entretenue et ses douches sont très belles. 
(30) als sie die augen wieder öffnet ist sie allein. der vertraute raum in dem sie sich befindet wirkt riesig, leer und bedrohlich.

Lorsqu'elle rouvre les yeux, elle est seule. La pièce où elle se trouve, qui lui est pourtant familière, lui fait l'effet d'un espace immense, vide et menaçant.

(31) Die Raumaufteilung ist in etwa so, daß alle Räume in etwa gleich groß sind: Das Schlafzimmer ist sehr groß, das Bad ist sehr groß und hat große Spiegel, die geputzt werden wollen; die Küche ist groß und wohnlich; das Wohnzimmer wirkt eher leer, ist aber schön eingerichtet mit Möbeln, die man als modern bezeichnen kann ...

La distribution des pièces est à peu de choses près ainsi faite que toutes les pièces sont à peu de choses près également grandes : la chambre à coucher est très grande, la salle de bain est très grande et dispose de grands miroirs qui ne demandent qu'à être nettoyés; la cuisine est grande et logeable; le séjour fait plutôt vide, mais est bien aménagé, orné qu'il est de meubles que l'on peut qualifier de modernes...

(32) Das schreckt mich gar nicht ab, denn die blanke halle wirkt sauber und geraeumig und verheisst ebensolche zimmer.

Cela ne me rebute en rien, car le hall d'entrée, dénué de tout ornement, fait propre et spacieux, et promet des chambres qui lui ressemblent.

Mention spéciale pour l'exemple (30), qui donne on ne peut plus explicitement le regard, et plus précisément les yeux (die augen), de l'observateur - de l'observatrice en l'occurrence - comme le filtre à travers lequel l'espace familier (der vertraute raum) a pu produire sur elle, paradoxalement, l'effet d'un espace immense, vide et menaçant (riesig, leer und bedrohlich); il importe cependant de souligner que ce regard de l'observateur est ici mentionné dans le contexte, et non dans la relation prédicative elle-même, autrement dit : «wirken» n'est certes pas incompatible avec le regard de l'observateur, mais «wirken» lui-même ne contient pas l'œil !

\subsection{Aussehen}

Par comparaison, c'est bien à travers le regard - à travers l'œil - de l'observateur extérieur que «aussehen» va décrire l'aspect (extérieur) de la pièce. En voici un exemple : l'observateur est sur le point de quitter la maison qui l'a accueilli, il est dans sa chambre, tout est emballé. Tout ? Oui, tout. Il regarde, dit-il, une dernière fois ces lieux qui lui sont devenus familiers : «Ein letztes Mal das Altvertraute sehen»-j'insiste sur ce «sehen», car c'est l'observateur / énonciateur qui le dit, ce n'est pas moi. Exemple (33):

(33) Gegen 3 Uhr nachts nach Hause. Im Haus ist alles still. Alle schlafen. Mein Zimmer sieht unheimlich leer aus. Die Wände kahl. Die Koffer gepackt, mitten im Zimmer. Letzte Überlegungen - alles eingepackt? Ja. Ich sitze auf der Bettkante und denke einfach nur nach. «Ein letztes Mal das Altvertraute sehen ...»

Je rentre à la maison sur le coup de trois heures du matin. Tout est calme. Tout le monde dort. Ma chambre a l'air vide à faire peur. Les murs sont nus, les valises bouclées, au beau milieu de la chambre. Ultimes vérifications : toutes mes affaires sont-elles bien dans les valises? Oui. Je suis assis sur le bord de mon lit, plongé dans mes pensées. «Voir une dernière fois ces lieux qui me sont devenus familiers...”

19 Pour essayer de comprendre le jeu de la base complexe «aussehen», peu analysable en synchronie, mais soumise aux variations de sens imposées par la variation des constructions («leer aussehen» / avoir l'air vide-«nach jemandem aussehen» / chercher quelqu'un du regard ou des yeux), il faut se souvenir sur le plan historique du fait que «sehen», comme l'infinitif germanique, est diathétiquement neutre et signifie aussi bien «voir» que "être vu, paraître», comme a pu le souligner Jacob Grimm au xix ${ }^{e}$ siècle : «videre»-«videri». Quant à «aus», il désigne simplement une vectorialisation, avec comme point d'origine un lieu pragmatiquement présenté comme «intérieur» ou «zone de 
contact extérieur» et comme point de direction un lieu présenté comme se situant dans la partie antérieure relative d'un repère (communication Yvon Desportes, 14 juin 2005). Aussi la relation entre l'observateur qui regarde, qui voit, et l'objet de l'observation d'où émane un certain aspect extérieur est-elle éminemment duelle, double, des deux côtés : d'une part, l'objet de l'observation est source / origine (aus) en même temps qu'il est vu par l'observateur (sehen) ; d'autre part, l'observateur regarde / voit l'objet (sehen) et reçoit quelque chose qui lui est envoyé par ledit objet (aus), l'observateur pouvant alors apparaitre comme le destinataire, le but de l'envoi en question. On comprend qu'il n'y ait dans ces conditions aucune place pour un datif «mir» siège d'un quelconque procès.

\subsection{Wirken et aussehen}

C'est ainsi la définition de la source qui fait la différence entre «wirken» et «aussehen» :

- avec «wirken», la source c'est l'objet, qui fait un effet sur moi, suscite une impression, bref crée une «apparence» : les propriétés physiques de la pièce font que je la trouve vide ; l'effet produit a l'objet comme terme-source et l'observateur comme terme-but ; l'énonciateur est à la fois récepteur (but de la lexis) et constructeur (évaluateur) ;

- avec «aussehen», la véritable source est le voyeur qui regarde un but-objet extérieur à lui la source de «sehen» ne peut être que l'énonciateur : ich sehe das Zimmer, je suis le regardeur, das Zimmer sieht leer aus, la pièce ne regarde rien, elle ne «regarde» que parce que «je» la regarde.

L'énonciateur reste implicite dans les deux cas, car il n'est pas en position de source de la relation prédicative. À cet égard, on comparera ces deux expressions de l'apparence aux expressions du genre : «Ich finde, dass..., ich sehe, dass...»

\subsection{Scheinen}

\subsection{1. «Scheinen»}

«Scheinen» - qui est celui de nos quatre noyaux verbaux qui a le plus souvent retenu l'attention des chercheurs, comme le montrent les éléments de bibliographie annexés à cet article - dit l'apparence opposée à l'essence, à l'être. Toujours Google, exemple (34) : à Monstropolis, à l'heure de la fermeture, le monstre Sulli aperçoit une porte - une de ces portes de placard qui permettent d'accéder au monde des humains - encore en fonction. Question : comment est-ce possible ? Un monstre serait-il encore au travail ? Première phase de son observation : selon toute apparence (schein-), la pièce est vide. Deuxième phase de son observation : la pièce est («ist», c'est-à-dire «sei-», c'est-à-dire l'être, ce «ist» étant d'ailleurs dans cet enchaînement clairement porteur d'un accent contrastif) effectivement vide, pour la simple raison que l'enfant qui s'y trouvait un instant auparavant s'est accroché au pauvre Sulli et ne le lâche plus! L'exemple (35) est moins explicite sur la deuxième phase des opérations : selon toute apparence, la cartouche d'hydrogène prélevée sur un poste d'incendie dans une phase précédente de jeu - car il s'agit d'un jeu sur ordinateur - ne contient pas tout le gaz qu'elle devrait, il va falloir refaire le plein : le joueur doit trouver le lieu où peut se faire l'opération! Dans ce deuxième exemple, la deuxième phase des opérations aurait pu consister à constater que la cartouche est effectivement vide, mais le jeu en fait l'économie et, se contentant de la seule «apparence», envoie de toute façon le joueur à la recherche des moyens de la remplir - ce qui est une autre façon d'affirmer la vacuité effective, au moins partielle, ne 
serait-ce que pour les besoins du jeu, de la cartouche en question! Voici les deux exemples :

(34) Neugierig schaut Sulley durch die Tür. Vielleicht ist hier noch ein Monster bei der Arbeit? Aber das Zimmer scheint leer. Und es ist tatsächlich leer, denn das kleine Mädchen (Kim und Maya McMahon), das eben noch im Bett lag, hat sich an Sulleys zotteliges Rückenfell geklammert und gelangt auf diese Weise in die Monsterwelt. Sulley ist außer sich: Er will das hochgiftige Geschöpf unbedingt loswerden, doch was er auch versucht, das Mädchen lässt ihn einfach nicht los.

Sulli, curieux, regarde par la porte. Un monstre serait-il peut-être encore au travail à cette heure? Mais la chambre semble vide. Et elle l'est véritablement, car la petite fille qui y dormait il y a un instant à peine s'est accrochée à la longue fourrure du dos de Sulli et a pu ainsi entrer au pays des monstres. Sulli, fou de rage, cherche à se débarrasser par tous les moyens de cette créature au venin mortel, mais ses efforts sont vains, la petite fille ne le lâche pas.

(35) Wende Dich dem Roboter zu und setze die Wasserstoffpatrone aus der Feuerwache hier ein. Sie scheint leer zu sein. Also nimmst Du sie wieder heraus, um sie irgendwo zu füllen.

Tourne-toi vers le robot et introduis la cartouche d'hydrogène que tu as précédemment prélevée sur le poste d'incendie. Elle semble vide. Tu la retires donc à nouveau et vas la remplir là où tu pourras.

\subsection{2. «Scheinen» et «aussehen».}

Il devient alors possible de préciser au passage la nature de l'opposition entre «scheinen» et «aussehen» : «scheinen» est totalement imprégné de la présence d'un être pensant qui, cherchant à appréhender la réalité du monde, essaye à tout moment de formuler des hypothèses, même si celles-ci ne sont encore qu'au stade de leur élaboration ; «aussehen» fournit quant à lui des résultats d'observation sur lesquels peut s'appuyer, entre autres, la formulation desdites hypothèses. C'est ainsi que le médecin attaché à la personne du prince et de la princesse régnant sur la petite cour où E. T. A. Hoffmann fait arriver le moine Médard au cours de son errance formule son hypothèse sur l'état de santé dudit moine - lequel suscite tant d'interrogations diverses par son comportement étrange à bien des égards - à l'aide de «scheinen», s'appuyant ensuite entre autres, pour fonder son diagnostic, sur le critère fourni par l'observation du personnage dans son aspect extérieur à l'aide de «aussehen» (Die Elixiere des Teufels, II, 1) :

(36) Der Leibarzt änderte plötzlich Blick und Ton. «Sie scheinenmir», sprach er milde und ernst, «Sie scheinen mir aber doch wirklich krank. - Sie sehen blaß und verstört aus - Ihr Auge ist eingefallen und brennt seltsam in rötlicher Glut ... Ihr Puls geht fieberhaft ... Ihre Sprache klingt dumpf ... soll ich Ihnen etwas aufschreiben?» - «Gift!», sprach ich kaum vernehmbar. - «Hoho!» rief der Leibarzt, «steht es so mit Ihnen? Nun, nun, statt des Gifts das niederschlagende Mittel zerstreuender Gesellschaft. - Es kann auch sein, daß ... Wunderlich ist es aber doch ... vielleicht -» «Ich bitte Sie, mein Herr!» rief ich ganz erzürnt, «ich bitte Sie, mich nicht mit abgebrochenen unverständlichen Reden zu quälen, sondern lieber geradezu alles ...» «Halt!» unterbrach mich der Leibarzt, «halt ... es gibt die wunderlichsten Täuschungen, mein Herr Leonhard; beinahe ist's mir gewiß, daß man auf augenblicklichen Eindruck eine Hypothese gebaut hat, die vielleicht in wenigen Minuten in nichts zerfällt. Dort kommt die Fürstin ...»

Le médecin changea brusquement de ton et de regard.

"Vous me semblez», dit-il d'une voix douce et grave à la fois, «vous me semblez tout de même, il faut que je vous le dise, vraiment malade : vous avez la mine blême d'un homme tourmenté, vos yeux sont enfoncés et brillent d'une rougeur étrange, vous avez le pouls fiévreux, vous parlez d'une voix sourde, voulez-vous que je vous prescrive quelque chose?» 
«Du poison!» dis-je d'une voix à peine audible.

«Oh !» s'écria le médecin, "vous en êtes rendu là? Je vous propose dans ce cas en fait de poison un médicament radical : la compagnie de personnes agréables et distrayantes. - Il peut également se faire que... Mais il y a quand même quelque chose d'étonnant... peut-être que...»

«Je vous en prie, Monsieur !», m'écriai-je au comble de la colère, «voulez-vous bien cesser de me tourmenter avec vos bribes de propos incompréhensibles, dites-moi bien plutôt tout ce que...»

Le médecin m'interrompit brusquement :

«Stop, on s'arrête là, les illusions des sens, ça existe, Monsieur Léonard, et les plus insidieuses : je suis à peu près certain que l'hypothèse que nous venons d'élaborer est fondée sur la seule impression du moment et qu'elle va s'écrouler peut-être dans les minutes qui viennent. Mais voilà la princesse qui approche...»

«Aussehen» ne fournit dans cette affaire qu'un critère lié à l'observation, non le moindre certes puisqu'il arrive en première place, mais d'autres critères d'appréciation contribuent à l'élaboration du diagnostic du médecin : l'observation de l'œil, la mesure du pouls, la prise en compte du langage. On voit très clairement la pensée scientifique à l'œuvre dans cet exemple, aux prises il est vrai avec des phénomènes supra-naturels, puisque rien moins que le Diable lui-même est mis en scène par le truchement du moine, et le médecin fait tous ses efforts pour signaler à son patient improvisé que son diagnostic est un diagnostic provisoire, un diagnostic - comme je l'ai précédemment esquissé - en attente de confirmation ou d'infirmation : «es kann auch sein», «wunderlich ist es aber doch», «vielleicht», pour ne rien dire du point culminant représenté par l'énoncé final, véritable définition de «scheinen» et démonstration éclatante d'une prudence éminemment scientifique, consciente qu'elle est d'être fondée peut-être sur «la seule impression du moment»! On comprend alors la possibilité - je dis bien la possibilité et non la nécessité - d'un datif «mir», point de réception de ladite impression et siège de l'ensemble du processus d'élaboration.

\subsubsection{Apparence, modalisation et report d'assertion.}

Il n'est pas impossible que nous tenions là également un critère permettant de distinguer nettement «scheinen» des verbes de «modalité» chers à Gabriele Diewald $(2000,334)$ : apparence / hypothèse, degré de probabilité - et il convient à ce titre d'ajouter à sa liste «könnte», et «wird» qui, s'il n'est certes pas un verbe de «modalité», est un verbe de «modalisation» au sens de Marcel Pérennec - et report d'assertion - pour «soll» et «will» (Diewald 2001, $89 ; 2004,242$ ) qui renvoient la responsabilité d'une assertion à un autre que le producteur de l'énoncé qui les contient - ne sont pas la même chose. Et en effet, les conditions de production des énoncés «Hans muss krank sein» et «Hans scheint krank zu sein» par exemple sont pour le moins fort différentes. Là où je dis «Hans muss krank sein» parce que Jean n'est pas là et que je suppose que c'est, que ce ne peut être que parce qu'il est malade, rien à voir avec «scheinen» qui présuppose la présence de l'objet d'observation ou tout au moins, en l'absence de «contact visuel» (Diewald 2000, 337), la prise en compte de ce qu'on peut en percevoir ou en savoir. Exemples :

Présence de l'objet d'observation, exemple (37) :

(37) Ich habe in meinem Garten eine Taube gefunden, die immer fast einschläft. Ich habe ihr ein bisschen Futter gegeben (Körner), aber sie hat es gefressen, plustert sich jetzt auf und scheint immer fast einzuschlafen. Die Taube hat einen gelben Ring mit einer 11 drauf und einen grünen Ring am anderen Fuß. Die Taube scheint krank zu sein, sie kackt mir den ganzen Garten voll. Wenn die Taube jetzt nicht 
wegfliegt, wird sie heut nacht von der Katze erwischt, und einen Käfig hab ich nicht ...

J'ai trouvé dans mon jardin un pigeon qui est en permanence dans un état proche de la léthargie. Je lui ai donné un peu de nourriture (des grains), mais il l'a mangée, hérisse en ce moment même ses plumes et a pour autant toujours un air proche de la léthargie. Ce pigeon a un anneau jaune marqué du nombre 11 et un anneau vert à l'autre patte. Ce pigeon semble bien malade, mon jardin déborde de ses déjections. S'il ne prend pas maintenant son envol, le chat ne le ratera pas cette nuit, et je n'ai pas de cage en ma possession...

Absence de contact visuel, exemples (38) et (39) :

(38) In diesem Buch [Aus der Bahn geworfen, Der jüdische Theatermann Dr. Hans Kaufmann, von Manfred Fuhrmann, Bielefeld, Aisthesis Verlag, 2003] geht es doch weniger um Berühmtheiten, im Gegenteil. Es ist zum Teil ein geradezu archäologisches Buch, in dem unbekannte Namen ausgegraben werden, um der vielschichtigen Theatergeschichte des zwanzigsten Jahrhunderts eine vergessene Facette zurückzugeben. Hans Kaufmann scheint ein experimentierfreudiger Praktiker gewesen zu sein, dem es Spaß machte, Neues zu entdecken, Stücke uroder erstaufzuführen und dabei auch mal Risiken einzugehen. [...] Und dann gibt es noch einen anderen Aspekt. Hans Kaufmann war Jude [...].

Ce livre ne traite pas de personnages célèbres, tout au contraire. C'est pour une part un livre littéralement archéologique, où l'on exhume des noms inconnus dans le but de restituer, de reconstituer une facette oubliée de l'histoire si riche, si composite du théâtre du XXe siècle. Hans Kaufmann semble avoir été un praticien toujours prêt à de nouvelles expériences, prenant plaisir à découvrir des talents nouveaux, à mettre en scène des œuvres originales ou à représenter des ceuvres qui ne l'avaient jamais été auparavant, prenant au passage de gros risques. [...] Et puis il y a encore un autre aspect du personnage : Hans Kaufmann était juif [...].

(39) (Spätestens) 1783 hat K. [Kottwitz, Hans Ernst, Baron von] dann Charlotte Helene Gräfin von Zedlitz (*12.6.1756) geheiratet. Die Ehe scheint nicht besonders glücklich gewesen zu sein. Immerhin entstammen ihr 5 Kinder ...

En 1783 au plus tard, le baron de Kottwitz a épousé en secondes noces Charlotte Hélène comtesse de Zedlitz, née le 12 juin 1756. Ce mariage ne semble pas avoir été particulièrement heureux. Toujours est-il que cinq enfants naîtront de cette union...

Etc.

Un autre test qui distinguera sans équivoque les tournures avec «scheinen» desdits verbes de modalisation ou de report d'assertion est la présence possible d'un datif («mir», etc.) dans les contextes de «scheinen» et son impossibilité absolue dans ceux des autres verbes en question : *Hans muss / wird / dürfte / kann / könnte / mag / soll / will mir krank sein . Dans un contexte de ce genre, contenant un tel datif, il est clair que la commutation de «scheinen» avec ces autres verbes est rigoureusement impossible.

\section{5. «Erscheinen»}

«Erscheinen», pour finir, ne décrit pas tant l'apparence que le résultat d'un processus : il s'est passé quelque chose, quelqu'un que l'on s'attendait à trouver là n'est PAS là, n'est PLUS là. Ce n'est pas une apparence, c'est la réalité - terrible en l'occurrence - à laquelle est confronté le personnage - féminin dans l'exemple (40) ci-après - décrivant les sentiments qui l'animent : «Sein Zimmer erscheint mir leer und fremd.» N'est-ce pas tout à fait extraordinaire? «Erscheinen» comme le résultat d'un processus, et du coup : c'est l'état - aussi paradoxal que cela paraisse, si j'ose dire, et l'on ne manquera pas de remarquer dans le contexte suivant l'emploi du verbe «ist» - de la pièce qui est décrit ! Avec juste une petite nuance : tout se passe à travers le regard du locuteur, d'un locuteur 
au datif (mir), siège du procès - et ce n'est pas par hasard, car la personne est, face à cette situation, dans l'incapacité de poser aucun acte. Voici l'exemple, que me fournit encore Google :

(40) Ich sitze hier in seinem Zimmer, auf seinem Bett und fühle mich genauso leer und fremd, wie sein Zimmer mir erscheint. Es ist kaum zu glauben, dass er gestern noch hier lebte. Das Zimmer hat überhaupt nichts mehr mit ihm zu tun. Es ist ein großer leerer Raum und es ist kalt.

Je suis dans sa chambre, je suis assise sur son lit, et je me sens vide et étrangère, tout comme sa chambre m'apparait vide et étrangère. Comment pourrait-on croire qu'il vivait là hier encore ! La chambre n'a absolument plus rien à voir avec lui. C'est une grande pièce vide, où il fait froid.

Le résultat d'un processus tel que le réalise «erscheinen» peut encore être illustré par l'exemple (41) ci-dessous, issu du domaine médical, qui expose par quels troubles se révèle cette affection particulière de la rétine qu'est la dégénérescence maculaire sénile : distorsion des lignes droites ou vide total au centre du champ visuel sont en effet le stade ultime de développement de cette affection. Et «erscheinen» vient alors marquer le décalage, d'ordre pathologique, qui s'est instauré entre les lignes qui sont dans la réalité bel et bien droites - la question ne se pose pas de savoir ici s'il serait quelque part possible qu'elles ne le soient pas, la référence est bien la vision normale que peut en avoir un œil non atteint par cette altération de la vision - et la perception qu'en a le malade qui souffre de cette affection. Le décalage également entre le vide visuel qui est le lot du malade atteint de cette affection et l'image qui se forme normalement au centre de la rétine d'un œil sain. On remarquera avec intérêt dans ce même extrait, entre les deux emplois de "erscheinen», l'expression de l'atténuation d'un effet produit, à l'aide de «wirken» et du comparatif «blasser», et celle de la constatation d'un état de fait à l'aide de «sein» - nous sommes en effet au terme d'un processus :

(41) Symptome der altersabhängigen Makuladegeneration.

Die angegebenen Beschwerden können sehr unterschiedlich sein. Manchmal wird nur ein Auge betroffen, während das andere für viele Jahre gut sieht. Mögliche Symptome:

1. Gerade Linien erscheinen verbogen, z.B. ein Fensterrahmen («Verzerrtsehen»).

2. Die Farben wirken blasser.

3. Worte auf einer Schriftseite sind verschwommen.

4. Das Zentrum des Gesichtsfeldes erscheint leer oder als grauer Fleck. Symptômes de la dégénérescence maculaire sénile.

Les troubles qui affectent la zone centrale de la rétine peuvent être très variables. C'est le plus souvent la vision d'un seul ceil qui se trouve altérée, tandis que l'autre œil pourra conserver des années durant une vision correcte. Symptômes possibles:

1. Déformation des lignes droites, un châssis de fenêtre par exemple paraîtra tordu.

2. Dépigmentation des couleurs.

3. Vision floue des caractères imprimés.

4. Perte de la vision centrale ou perception centrale réduite à une tache grisâtre.

\section{Bilan}

31 Tel est en allemand, rapidement présenté, le petit système clos de l'apparence, un système à quatre noyaux verbaux - wirken, aussehen, scheinen, erscheinen - dont le tableau suivant résume les caractéristiques essentielles. Confirmant la bipartition qui a pu être établie à l'aide de quelques tests, il laisse nettement apparaitre ce que je désignerais volontiers comme le camp des «pro-mir» (scheinen / erscheinen) et celui des 
«anti-mir» (wirken / aussehen). Dans le camp des «pro-mir», «scheinen» pose pleinement un énonciateur qui pense, qui raisonne ou qui spécule en marquant une distance entre son propos et le réel. Dans le camp des «anti-mir», c'est avec «aussehen» un énonciateur qui perçoit, et c'est avec «wirken» le récepteur d'une impression. Perception et spéculation constituent ainsi deux axes par rapport auxquels pourrait se définir également tel ou tel élément lexical dérivé à partir de ces noyaux verbaux : c'est bien la question du rapport à la «réalité» que posent des éléments tels que «wirklich» «vraiment» - en venant appuyer ou confirmer une information précédente, et «in Wirklichkeit» - «en réalité» - qui sert à relativiser ou à rectifier l'information ; un élément tel que «wahrscheinlich», qui ouvre le champ de la "vrai-semblance», est propre à exprimer la probabilité ; «scheinbar» - «en apparence» - et «anscheinend» «apparemment» - annoncent quant à eux un jugement fondé sur les apparences.

\begin{tabular}{|l|l|l|}
\hline & Propriétés syntaxiques & Valeur sémantique \\
\hline Wirken & Objet source & Production d'un effet \\
\hline Aussehen & $\begin{array}{l}\text { Énonciateur } \\
\text { source }\end{array}$ & Aspect extérieur \\
\hline Scheinen & $\begin{array}{l}\text { Énonciateur } \\
\text { but }\end{array}$ & $\begin{array}{l}\text { Apparence } \\
1^{\text {er }} \text { temps }\end{array}$ \\
\hline Erscheinen & Énonciateur & Etat \\
$2^{\text {nd }}$ temps
\end{tabular}

\section{BIBLIOGRAPHIE}

Askedal, John Ole 1983. « Über scheinen als Modalitätsverb im gegenwärtigen Deutsch ». In Askedal, John Ole / Leirbukt, Oddleif / Findreng, Ådne / Christensen, Ch. (Hrsg.), Festschrift für Laurits Saltveit zum 70. Geburtstag am 31. Dezember 1983. Bergen, Oslo, Tromsö: Universitetsforlaget. S. 30-39.

Askedal, John Ole 1998. « Satzmustervariation und Hilfsverbproblematik beim deutschen Verb scheinen ». In Donhauser, Karin / Eichinger, Ludwig M. (Hrsg.), Deutsche Grammatik: Thema in Variationen; Festschrift für Hans-Werner Eroms zum 60. Geburtstag. Heidelberg: Winter. S. 49-74.

Askedal, John Ole 2004. « Die Konstitution deutscher und norwegischer supinischer Verbketten in kontrastivem Vergleich. Zur Syntagmatik von Modalität und anderen Prädikatskategorien ». In Leirbukt, Oddleif (Hrsg.), Tempus / Temporalität und Modus / Modalität im Sprachvergleich. Tübingen: Stauffenburg Verlag (Eurogermanistik 18). S. 25-61. 
Baudot, Daniel 1989. Le problème de la voix et sa solution dans la langue allemande. Thèse de doctorat sous la dir. de François Schanen, université de Montpellier III.

Confais, Jean-Paul / Schanen, François 1986. Grammaire de l'allemand, formes et fonctions. Paris, Nathan.

Desportes, Yvon 1986. La préverbation en allemand au IX $X^{e}$ siècle. Thèse de doctorat d'État sous la dir. de Philippe Marcq, université de Paris IV.

Desportes, Yvon 1998. Les préverbes et la préverbation en allemand au IX $X^{e}$ siècle. 4 vols. Lyon, université de Lyon III-Jean-Moulin.

Diewald, Gabriele 2000. « Scheinen als Faktizitätsmarker ». In Habermann, Mechthild / Naumann, Bernd / Müller, Peter O. (Hrsg.), Wortschatz und Orthographie in Geschichte und Gegenwart. Festschrift für Horst Haider Munske zum 65. Geburtstag. Tübingen: Niemeyer. S. 333-356.

Diewald, Gabriele 2001. « Scheinen-Probleme: Analogie, Konstruktionsmischung und die Sogwirkung aktiver Grammatikalisierungskanäle ». In Müller, Reimar / Reis, Marga (Hrsg.), Modalität und Modalverben im Deutschen. Hamburg: Buske. S. 87-111.

Diewald, Gabriele 2004. « Faktizität und Evidenzialität. Semantische Differenzierungen bei den Modal- und Modalitätsverben im Deutschen ». In Leirbukt, Oddleif (Hrsg.), Tempus / Temporalität und Modus / Modalität im Sprachvergleich. Tübingen: Stauffenburg Verlag (Eurogermanistik 18). S. 231-258.

Ebert, Robert Peter 1975. « Subject raising, the clause squish, and German scheinenconstructions ». In Grossman, Robin E. / San, L. James / Vance, Timothy J. (Hrsg.), Papers from the 11th regional meeting, Chicago Linguistic Society, April 18-20, 1975. Chicago: Chicago Linguistic Society. S. 177-187.

Grimm, Jacob und Wilhelm 1854. Deutsches Wörterbuch. Leipzig: Verlag von S. Hirzel. Bd. 1, Sp. 967, Art. Aussehen: «Aussehen, nnl. uitzien, wie das einfache sehen bald videre, bald videri bedeutet».

Lazard, Gilbert 1995. « Préverbes et typologie ». In Rousseau, André (éd.), Les préverbes dans les langues d'Europe, introduction à l'étude de la préverbation. Lille, Presses universitaires du Septentrion, pp. 23-31.

McKay, Terence 1985. Infinitival complements in German. Lassen, scheinen and the verbs of perception. Cambridge, usw.: Cambridge University Press (Cambridge Studies in Linguistics Supp.).

Meurers, Walt Detmar 2000. Lexical generalizations in the syntax of German non-finite constructions. Diss., Tübingen.

Olsen, Susan 1981. Problems of seem / scheinen-constructions and their implications for the theory of predicate sentential complementation. Tübingen: Niemeyer (Linguistische Arbeiten 96).

Pafel, Jürgen 1989. « scheinen + Infinitiv ». In Falkenberg, Gabriel (Hrsg.), Wissen, Wahrnehmen, Glauben. Epistemische Ausdrücke und propositionale Einstellungen. Tübingen: Niemeyer. S. 123-172.

Pérennec, Marcel 1974. « Modalisateurs et appréciatifs de l'allemand » In Linguistica Palatina 11.

Pérennec, Marcel 1979. Illocution et assertion en allemand. Thèse de doctorat d'État sous la dir. de Paul Valentin, université de Paris IV.

Richter, Michael 2002. «Scope and semimodal verbs ». In Hentschel, Elke (Hrsg.), Fremdsprachenlernen / Foreign language teaching. Online: http://www.linguistik-online.de/10_02/ richter. html. 
Rousseau, André 1995. «Fonctions et fonctionnement des préverbes en allemand. Une conception syntaxique des préverbes » In Rousseau, André (éd.), Les préverbes dans les langues d'Europe, introduction à l'étude de la préverbation. Lille, Presses universitaires du Septentrion, $\mathrm{p}$. 23-31.

Seppänen, Aimo / Hall, Christopher 1984. « Es scheint uns, dass es so ist. Zur Syntax von scheinen im heutigen Deutsch ». In Festschrift für Lauri Seppänen zum 60. Geburtstag. Tampere: Universität. S. $129-141$

Solatie, Raija 1996. « Verbes psychologiques dans une construction impersonnelle. Analyse contrastive allemand-français » In Nouveaux Cahiers d'allemand n 14, pp. 421-436.

Vassileva, Rada 2004. «Zu einem besonderen Gebrauch des Verbs scheinen ». In Stanescu, Speranta (Hrsg.), Die Valenztheorie. Bestandaufnahme und Perspektiven. Dokumentation einer wissenschaftlichen Tagung in Sibiu / Hermannstadt im Februar 2002. Frankfurt am Main u.a.: Lang. S. $165-170$.

\section{Sources des exemples utilisés}

Dictionnaire français-allemand Harrap's Weis Mattutat 1989. Londres, Paris, Stuttgart, Klett Verlag,.

Duden Deutsches Universalwörterbuch A-Z 1989, hrsg. und bearb. vom Wiss. Rat u. d. Mitarb. d. Dudenred. unter Leitung von Günther Drosdowski, 2. Aufl., Mannheim, Wien, Zürich, Dudenverlag,.

Wörterbuch der deutschen Gegenwartssprache 1980-1981, hrsg. von Ruth Klappenbach und Wolfgang Steinitz, Berlin, Akademie-Verlag,.

Divers sites internet via Google, y compris l'exemple extrait de Die Elixiere des Teufels, de E. T. A. Hoffmann. Notre aimable lecteur y aura facilement accès en tapant dans Google tout ou partie de l'exemple concerné.

\section{RÉSUMÉS}

Dans le domaine germanique, la commutation fait apparaitre quatre noyaux verbaux : wirken, aussehen, scheinen et erscheinen. Ils constituent un petit système clos de l'apparence, au sein duquel ils partagent deux structures syntaxiques : la mise en relation avec une qualité et la mise en relation avec « als ob + groupe verbal ». Leur compatibilité ou incompatibilité avec un datif tel que « mir ", avec un complexe infinitival ou avec un groupe verbal introduit par « dass » amène à distinguer l'effet produit (wirken), la perception d'un énonciateur (aussehen), la distance que ce dernier établit entre son propos et le réel (scheinen), et le résultat d'un processus (erscheinen).

In German, four verbs are to be found : wirken, aussehen, scheinen and erscheinen. They make up a small closed system expressing the idea of appearance in which they share two distinctive syntactic structures : a qualitative construction and the «als ob + Verb Phrase» construction. Their compatibility or their incompatibility with a marker of the dative such as mir, with an infinitival cluster or with a verbal group introduced by dass leads us to make four semantic distinctions : the effect produced by the verb (wirken), the utterer's perception (aussehen), the gap set by the utterer between his discourse and the world (sheinen) and the result of a process ( erscheinen). 
INDEX

Mots-clés : apparence, noyaux verbaux, énonciateur extérieur, observateur, perception, système clos

\section{AUTEUR}

ALAIN CAMBOURIAN

Université de Tours 